\section{Check for updates}

Cite this: J. Mater. Chem. B, 2019, 7, 3320

DOI: $10.1039 / c 9 t b 90064 k$

rsc.li/materials-b

\title{
Correction: Synergy effects of copper and silicon ions on stimulation of vascularization by copper-doped calcium silicate
}

\author{
Ni Kong, ${ }^{a}$ Kaili Lin, ${ }^{b}$ Haiyan Li*a and Jiang Chang*ab
}

Correction for 'Synergy effects of copper and silicon ions on stimulation of vascularization by copperdoped calcium silicate' by Haiyan Li, Jiang Chang et al., J. Mater. Chem. B, 2014, 2, 1100-1110.

The authors regret that the incorrect images were displayed for $\mathrm{Cu} 1 / 64$, CS 1/128 and $\mathrm{Cu} 0.3$ in Fig. 4 of the original version of this manuscript. The correct version of Fig. 4 is shown below. The caption remains unchanged.
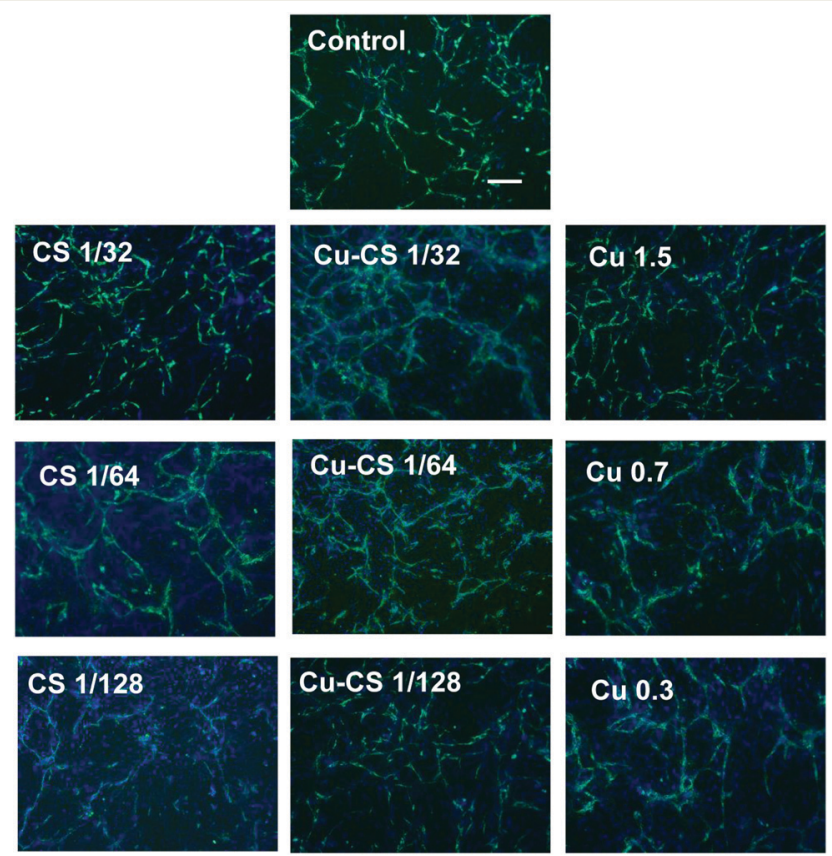

Fig. 4 Tubule formation by co-HUVECs in direct contact co-cultures was stimulated by CS and Cu-CS extracts diluted with control medium at $1 / 32$, $1 / 64$ and 1/128, which is indicated by the VWF stained co-HUVECs and statistical study at the end of the cultures (7 days) (a). Immunofluorescence staining of VWF is in green and nuclei in blue. Nuclei were stained in blue with DAPI. Bar $=200 \mu \mathrm{m}$. (b) The quantification and statistical analysis of tubule formation was obtained by counting and comparing the number of tubes in the images. * and ** represent $P<0.05$ and $P<0.01$, respectively, when compared with control. \# indicates that the co-HUVECs cultured with Cu-CS extracts formed more tubes than those cultured with CS extracts at the same dilution ratios. (a indicates that the co-HUVECs cultured with $\mathrm{Cu}-\mathrm{CS}$ extracts at the dilution ratio of $1 / 32$ formed more tubes than those cultured with CS extracts at the dilution ratio of $1 / 128$ although these two extracts contain the same concentrations of Si ions.

The Royal Society of Chemistry apologises for these errors and any consequent inconvenience to authors and readers.

\footnotetext{
${ }^{a}$ Med-X Research Institute, School of Biomedical Engineering, Shanghai Jiaotong University, 1954 HuaShan Road, Shanghai 200030, China. E-mail: haiyan.li@sjtu.edu.cn; Fax: +86-21-62933243; Tel: $+86-21-62933243$

${ }^{b}$ Shanghai Institute of Ceramics, Chinese Academy of Sciences, 1295 Dingxi Road, Shanghai 200050, China. E-mail: jchang@mail.sic.ac.cn; Fax: +86-21-52413903; Tel: $+86-21-52412804$
} 